\title{
Proper motions of thin filaments at the Galactic Center
}

\author{
K. Mužić, ${ }^{1}$ A. Eckart, ${ }^{1,2}$ R. Schödel, ${ }^{1}$ L. Meyer ${ }^{1}$ and A. Zensus ${ }^{2,1}$ \\ ${ }^{1}$ I. Physikalisches Institut, Universität zu Köln, Zülpicher Str. 77, 50937 Köln, Germany \\ ${ }^{2}$ Max-Planck-Institut für Radioastronomie, Auf dem Hügel 69, 53121 Bonn, Germany \\ email: muzic@ph1.uni-koeln.de
}

\begin{abstract}
We present the proper motion study of the thin filaments observed in L'-band $(3.8 \mu \mathrm{m})$ adaptive optics images of the central parsec of the Milky Way. Observed filaments are associated with the mini-spiral and, in some cases, with stars. They can be interpreted as shock fronts formed by the interaction of a central wind with the mini-spiral or extended dusty stellar envelopes.
\end{abstract}

Keywords. Galaxy: center - infrared: ISM

\section{Introduction}

The central cavity of the Galactic Center is surrounded by a dense clumpy molecular ring (also called circum-nuclear disk, CND) of warm dust (Zylka et al. 1995) and neutral gas (e.g., Güsten et al. 1987). The central cavity itself has a much lower mean gas density and contains the so called mini-spiral, which consists of mostly ionized gas and dust, and connects the CND to the center of the stellar cluster. Adaptive optics L'-band $(3.8 \mu \mathrm{m}$; Fig. 1, left) images of the central parsec of the Milky Way show large amounts of gas and dust belonging to the mini-spiral. Additionally, one can distinguish between a large number of thin filaments (some have already been reported by Clénet et al. 2004) Here we identify them and present proper motion measurements. We argue that they are most likely shock fronts formed in interaction of the strong winds with an ambient ISM.

Observations were performed using the NAOS/CONICA adaptive optics system at the ESO VLT. The data set includes L'-band images from 5 epochs (2002 to 2006). Data reduction and image transformations were performed using the DPUSER software for astronomical image analysis (T. Ott) and IDL routines. Proper motions of the thin filaments were determined using the cross-correlation technique.

\section{Identification and proper motions of the thin filaments}

In this report we present only the thin filaments for which we were able to determine the proper motions. The results obtained in our study are given in Table 1 . and shown in Fig. 1. Filaments labeled NE are located along the inner rim of the northern arm and are curved with their convex sides eastwards, same as the stellar bow-shock X1. Filaments to the west and southwest of Sgr A* are curved with their convex sides westward and are positioned almost perpendicular to the Bar (those labeled SW). Feature X7 shows a cometary structure and points approximately to $\operatorname{Sgr} \mathrm{A}^{*}$.

Our results support the model of a collimated outflow originating from the disk of young mass-losing stars around Sgr A* (e.g., Paumard et al. 2006) or, alternatively, from the massive black hole itself (for more details see Mužić et al. 2006). 


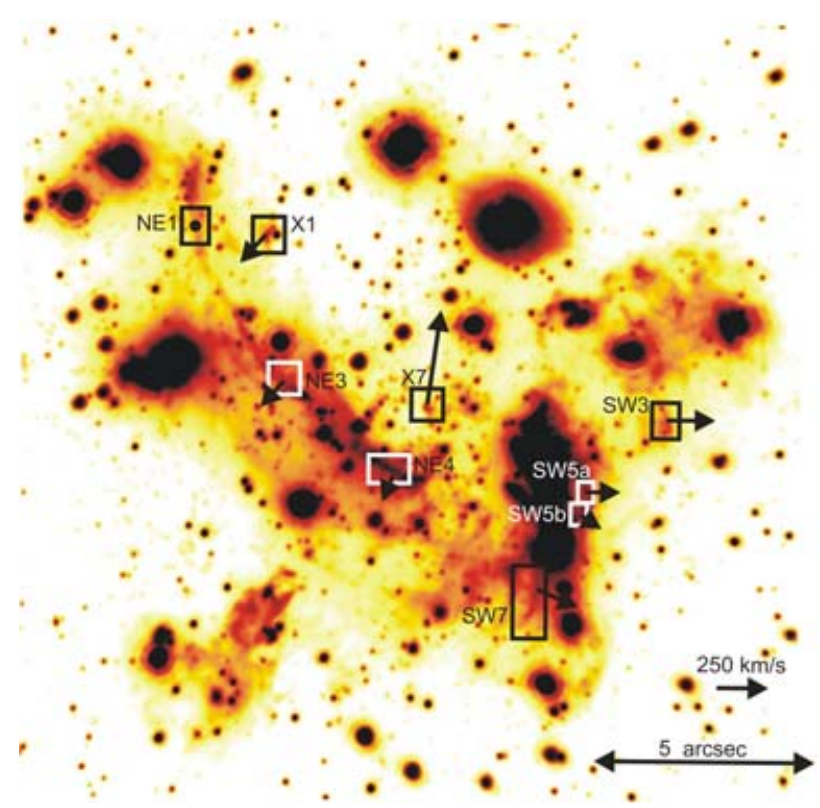

Figure 1. L'-band image of the Galactic Center. Boxes mark thin filaments with measurable proper motions. Note that boxes in this image are larger than those used for measurements (stated in Tab. 1). Arrows show proper motions of the thin filaments obtained in our study. Insignificant motion of the Northern Arm filament NE1 is marked with a circle.

Table 1. Proper motions of the thin filaments

\begin{tabular}{lccccc}
\hline \hline Feature & $\begin{array}{c}\Delta \alpha \\
(\operatorname{arcsec})\end{array}$ & $\begin{array}{c}\Delta \delta \dagger \\
(\operatorname{arcsec})\end{array}$ & $\begin{array}{c}\text { size }(\Delta x \times \Delta y) \\
(\operatorname{arcsec} \times \operatorname{arcsec})\end{array}$ & $\begin{array}{c}v \ddagger \\
(\mathrm{km} / \mathrm{s})\end{array}$ & $\begin{array}{c}\Delta v \\
(\mathrm{~km} / \mathrm{s})\end{array}$ \\
\hline NE1 & 4.76 & 3.42 & $0.32 \times 0.46$ & -29 & 16 \\
NE3 & 2.62 & -0.09 & $0.38 \times 0.41$ & 167 & 25 \\
NE4 & 0.32 & -1.95 & $0.43 \times 0.43$ & 130 & 18 \\
SW3 & -6.08 & -0.77 & $0.35 \times 0.35$ & -229 & 19 \\
SW5a & -4.20 & -2.31 & $0.35 \times 0.11$ & -165 & 15 \\
SW5b & -4.13 & -2.75 & $0.16 \times 0.24$ & -123 & 22 \\
SW7 (R.A.) & -3.07 & -5.10 & $0.73 \times 1.22$ & -186 & 17 \\
SW7 (Dec.) & -3.07 & -5.10 & $0.73 \times 1.22$ & -80 & 21 \\
X1 (R.A.) & 3.22 & 3.40 & $0.30 \times 0.38$ & 113 & 16 \\
X1 (Dec) & 3.22 & 3.40 & $0.30 \times 0.38$ & -78 & 21 \\
X7 (R.A.) & -0.56 & 0.55 & $0.54 \times 0.41$ & -71 & 17 \\
X7 (Dec) & -0.56 & 0.55 & $0.54 \times 0.41$ & 458 & 21 \\
\hline
\end{tabular}

$\dagger$ Relative to Sgr A*; position of the center of the box

$\ddagger$ Velocities of all NE and SW features, except that of SW7, are in a direction perpendicular to the shock feature. Other proper motions are given in both R.A. and Dec. direction.

\section{References}

Clénet, Y., Rouan, D., Gendron, E. et al. 2004, A\&A, 417, L15

Güsten, R., Genzel, R., Wright, M. C. H. et al. 1987, ApJ, 318, 124

Mužić, K., Eckart, A., Schödel, R. et al. 2006, submitted to A\&A

Paumard, T., Genzel, R., Martins, F. et al. 2006, ApJ, 643, 1011

Zylka, R., Mezger, P. G., Ward-Thompson, D. et al. 1995, A\&A, 297, 83 